\section{Cahiers de Narratologie}

Analyse et théorie narratives

$17 \mid 2009$

Stéréotype et narration littéraire

\title{
Le récit de la quête dans le roman américain postmoderne : entre version parodique et stéréotypie. L'exemple de John Barth
}

\section{Salwa Karoui-Elounelli}

\section{OpenEdition}

\section{Journals}

\section{Édition électronique}

URL : http://journals.openedition.org/narratologie/1192

DOI : 10.4000/narratologie.1192

ISSN : 1765-307X

Éditeur

LIRCES

\section{Référence électronique}

Salwa Karoui-Elounelli, « Le récit de la quête dans le roman américain postmoderne : entre version parodique et stéréotypie. L'exemple de John Barth », Cahiers de Narratologie [En ligne], 17| 2009, mis en ligne le 10 janvier 2011, consulté le 19 avril 2019. URL : http://journals.openedition.org/ narratologie/1192 ; DOI : 10.4000/narratologie.1192

Ce document a été généré automatiquement le 19 avril 2019.

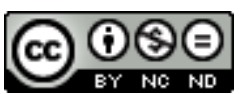

Cahiers de Narratologie - Analyse et théorie narratives est mis à disposition selon les termes de la licence Creative Commons Attribution - Pas d'Utilisation Commerciale - Pas de Modification 4.0 International. 


\title{
Le récit de la quête dans le roman américain postmoderne: entre version parodique et stéréotypie. L'exemple de John Barth
}

\author{
Salwa Karoui-Elounelli
}

«Where to ? It scarcely mattered.» John Barth, The Tidewater Tales

1 Le présent travail porte sur une question qui relève de la poétique du récit fictionnel postmoderne, à savoir sur la refonte soutenue de la narrativité romanesque par le biais d'une répétition subversive des motifs de la tradition picaresque et du roman de voyage. Souvent une forme paradoxale de stéréotype s'installe au cœur de l'esprit d'autocritique et d'auto-ironie qui anime une telle refonte et informe le déploiement des motifs conventionnels des traditions romanesques, notamment le motif de la quête.

2 En effet, l'examen métafictionnel du motif de la quête dans l'écriture romanesque postmoderne s'articule autour de ce que la tradition du roman picaresque a consacré comme réflexion critique sur la thématique du voyage ou de l'errance. Les modes de l'ironie et de la subversion qui canalisent une telle réflexion semblent promouvoir une image stéréotypée de la tradition picaresque, alors qu'ils reconnaissent en même temps la dépendance de la poétique du roman postmoderne à l'égard de l'autorité d'une telle tradition. D'où le caractère paradoxal de la notion même de stéréotype dans cette poétique. En outre, l'usage subversif et ironique du motif de la quête dans plusieurs romans américains postmodernes (usage qui assimile la tradition picaresque et le roman d'aventures au domaine du stéréotype) renforce souvent leur tendance métacritique, et accentue ainsi leur préoccupation d'une refonte de la poétique du genre romanesque. C'est une refonte qui met plus l'accent sur la dimension poétique que sur celle de la vraisemblance, et ce à travers une revalorisation du mode métaphorique de la narration dans l'écriture romanesque. 
3 En fait, le récit américain postmoderne entreprend sa réflexion critique sur le genre romanesque en reformulant les stratégies de la narration au sein du mode métaphorique et en maintenant un jeu intertextuel explicite. Certains romans, tels que The Crying of Lot 49 et Gravity's Rainbow de Thomas Pynchon, ou Death, Sleep and the Traveler, et Adventures in the Alaskan Skin Trade de John Hawkes, assimilent le mode métaphorique à une répétition parodique du motif de la quête et du thème du voyage, et c'est précisément ce qui leur permet d'attribuer à la tradition picaresque et au roman d'aventure un statut paradoxal oscillant entre le stéréotype et l'archétype. Les romans de John Barth depuis The Floating Opera (1956), notamment The TideWater Tales (1987) et The Last Voyage of Somebody the Sailor (1991) attestent le souci majeur de la première vague de la métafiction américaine, à savoir la nécessité de fonder une esthétique du roman qui articulerait sa signification et son contenu à une subversion permanente des traditions romanesques (le roman picaresque, le Bildungsroman et le roman d'aventures) et de leurs œuvres archétypales. Cette subversion se fait souvent à travers une révision critique d'un motif majeur de l'intrigue romanesque, notamment le motif de la quête. Depuis son premier roman, The Floating Opera (1956), Barth n'a cessé de revisiter les motifs de la quête et du voyage, mais aussi les métaphores du bateau et de la navigation, dans le dessein de mettre en évidence le caractère stéréotypé du récit de la quête ou de l'aventure maritime. Des romans tels que The TideWater Tales ou The Last Voyage of Somebody the Sailor joignent le ton de l'autoironie à une mise en question de la frontière même qui s'établit entre la consécration de l'aspect archétypal de certaines figures et textes littéraires et la production ou la consolidation des stéréotypes littéraires, notamment celui de l'aventure maritime, à travers la mise à nu de l'ancrage de tels stéréotypes dans des textes canoniques. L'autoironie qui engendre dans les récits fictionnels de Barth une perversion systématique du motif de la quête, débouche sur une mise en exergue de l'aspect stéréotypé qu'ont acquis les formes littéraires propres au récit romanesque, tels que le roman de voyage, le roman maritime, le récit de vie dans le roman de formation ou dans l'autobiographie fictionnelle, etc.

4 Le caractère métafictionnel des romans commentés dans le présent travail explique le fait que les stéréotypes qu'ils construisent sont des stéréotypes littéraires (ce qui n'implique guère leur indifférence aux stéréotypes culturels liés aux notions de race, de sexe, etc.). Dans les récits de Barth, la mise en exergue des stéréotypes littéraires émane surtout d'un processus de réflexion critique sur les conventions de la narration romanesque et sur les formes littéraires que l'écrivain postmoderne ne prétend pas pouvoir surpasser ou évincer. En effet, même si cette réflexion reconnaît le statut archétypal de certaines œuvres dans des traditions romanesques particulières (Don Quichotte dans la tradition picaresque, Robinson Crusoé ou l'île au trésor dans le roman d'aventures, etc.), la notion de stéréotype émerge souvent comme le fruit d'une subversion ironique de ces archétypes littéraires. La présence envahissante des œuvres littéraires ayant acquis le statut de textes canoniques (présence pressentie depuis l'époque romantique comme source d'anxiété1), est devenue, avec la littérature postmoderne, le signe de l'incertitude quant à la survie du genre romanesque. John Barth avait suggéré, dans un essai critique ${ }^{2}$ qui a fait date, que le romancier postmoderne puise sa propre thématique dans cette incertitude concernant l'avenir du genre. D'où le caractère foncièrement autoréflexif, voire autosubversif d'une partie majeure de la fiction américaine postmoderne. En outre, certains critiques attribuent cette tendance d'autosubversion à l'esprit même de la culture postmoderne dans son ensemble, à savoir, son caractère sceptique, ambigu et 
fragmentaire, un esprit qui transforme les archétypes en stéréotypes ${ }^{3}$. A l'encontre de la littérature moderniste (au moins dans le monde anglo-américain) qui évoque ou parodie les textes classiques dans un esprit d'hommage et de nostalgie, l'ironie subversive et la parodie propres à la fiction postmoderne ébranlent la notion même d'archétype, au point que celle-ci finit par développer sa poétique selon le mode de l'autodérision.

\section{De la typification au stéréotype littéraire dans l'écriture romanesque}

5 La notion de «type » dans les récits de fiction se rapporte généralement à la création de personnage et soulève presque toujours la question de la représentation, aussi bien quant à ses mécanismes qu’à sonesthétique.Ainsi, la poétique du roman réaliste devait se confronter à la question de l'équilibre entre la création d'un personnage-individu et celle d'un personnage-type «dont les traits seraient représentatifs d'une catégorie ou d'une classe sociale ${ }^{4} »$. Le croisement entre l'individualisation et la typification manifeste le lien viscéral entre la créativité et la vision synthétique dans l'écriture romanesque. Ce lien se traduit par le fait que les types littéraires sont créés, comme le décrit Chartier, "à l'articulation de l'observation et de la spéculation ${ }^{5}$ ». De même chez les premiers romanciers anglais, comme le suggère Ian Watt, la typification des personnages ne devait ni primer sur le souci du particulier, de l'individualisation, ni totalement disparaître'

Ainsi, l'émergence des types littéraires dans le domaine de la fiction, semble être le résultat inéluctable de cette oscillation que l'on perçoit dansles récits romanesques entre l'invention et la représentation, mais aussi entre la création et la répétition. Le personnage-type du "héros problématique ", né avec Don Quichotte et qui présente, d'après plusieurs lectures, une continuité ironique subversive avec le roman ("romance ») chevaleresque, semble indiquer l'enclavement de l'écriture romanesque depuis ses débuts dans un rapport vital (souvent paradoxal) avec les types littéraires qui émanent des genres classiques tels que l'épopée ou la comédie ${ }^{7}$. La poétique de la fiction postmoderne a été forgée par une telle appréhension du genre romanesque, et ce depuis ses débuts dans les années cinquante. Dans cette appréhension, les auteurs postmodernes se sont appliqués à se rendre attentifs à la tendance du roman à reprendre les composantes des genres classiques (tels que l'épopée, la comédie, le récit de chevalerie, etc.) en activant et épuisantleurs aspects "typiques" contre lesquels le roman postmoderne a mis en place ses propres types littéraires. La poétique postmoderne, surtout dans le roman américain, mise sur cette pluralité inhérente au roman afin de légitimer sa propre ambiguïté quant à ses rapports avec les formes et les textes littéraires qui ont régi l'écriture romanesque.

D'ailleurs, ce qui ressort des approches de l'histoire du roman c'est surtout le caractère intertextuel de celui-ci, son besoin de se définir par rapport aux traditions et aux textes littéraires du passé, par le biais de l'imitation parodique, de l'allusion, de la citation, etc. De telles pratiques intertextuelles impliquent souvent des interprétations qui varient dans leurs traitements du texte parodié ou cité, allant de la consécration de son statut de modèle (voire d'archétype, quand la parodie ou l'allusion révèle une attitude d'admiration envers l'intertexte) jusqu'à la répétition ridiculisant les thèmes et les personnages du texte parodié qui s'en trouvent transformés en stéréotypes littéraires. Ainsi, dans le roman anglais, la fiction de Henry Fielding attribue à Don Quichotte (à 
travers l'imitation et l'allusion) le statut d'archétype (dans Joseph Andrews) et re-présente, à travers la moquerie et le ridicule, les thèmes et les personnages de Pamela de Samuel Richardson comme des stéréotypes de l'arrivisme et de l'hypocrisie déguisés sous le masque de la vertu et de la chasteté.

Ce qu'on peut déduire des théories du roman, notamment celle de Bakhtin, c'est qu'il existe un rapport dynamique entre le mode de la parodie, souvent mentionné comme vital à l'évolution du genre romanesque ${ }^{8}$, et l'esprit satirique de la dimension carnavalesque $^{9} \mathrm{du}$ roman. Cette dimension, entendue dans sa portée figurative, est devenue l'image qui condense le fonctionnement du dialogisme romanesque et ses procédés de transgression des normes (sociales, politiques ou même littéraires ${ }^{10}$ ). L'axe de la parodie qui ancre l'écriture romanesque dans le monde de la discursivité et de l'intertextualité (d'où son autoconscience quasi omniprésente) peut renforcer et/ou dévoiler le stéréotypage qui émane souvent de l'image satirique créée par le côté carnavalesque du roman.

Mais le mode de la parodie instaure souvent dans l'écriture romanesque postmoderne un traitement complexe, voire à multiple facettes, du stéréotype littéraire. C'est à travers sa mise en question des possibilités même de la représentation littéraire que le récit parodique postmoderne suggère la notion de stéréotype, surtout quand sa portée métacritique suggère une assimilation des procédés de la représentation à ceux du stéréotypage. En insistant sur le lien paradoxal qui relie le stéréotype à la représentation (un lien allant de la fonction d'arrière-plan de la représentation à celle de sa négation même), Ruth Amossy décrit comme extrêmement relative la distance qui sépare l'effet réaliste (de la représentation) de la provocation du stéréotype ${ }^{11}$. La relativité, voire la précarité de cette distance se trouve mise à nue quand le récit métafictionnel postmoderne démystifie les procédés de la représentation en dévoilant l'aspect répétitif, conventionnel de la vraisemblance et de la plausibilité qui trop souvent ont réduit dans l'histoire du roman la portée mimétique du récit fictionnel aux structures figées des traditions romanesques ${ }^{12}$. De ce point de vue, le roman postmoderne assimile son esthétique de l'auto-ironie à une éthique de l'ouverture, de la pluralité et de l'insaisissable.

10 Mais le stéréotype semble aussi partager avec l'écriture parodique postmoderne une même relation dialectique avec l'acte de lecture. La lecture concrétise le stéréotype (comme elle concrétise le fonctionnement de la parodie) et est, à son tour, limitée par celui-ci à la «reconnaissance d'une structure répétitive ${ }^{13}$ ». Toutefois, la parodie n'engendre pas nécessairement cette réduction: le renvoi à d'autres textes (comme modèles) est un renvoi à d'autres 'terrains glissants' qui rapelle l'ouverture et la pluralité de l'interprétation. Même quand la répétition parodique a recours aux procédés d'une réduction caricaturale (qui est l'apanage du stéréotype) l'effet de stéréotypage qu'elle peut produire (en ironisant un type de personnage récurrent dans l'histoire du roman) incite le lecteur à porter un regard distant et critique sur les modèles littéraires qui ont été épuisés dans les traditions romanesques. Alors que la « lecture stéréotypée » (celle qui reconnaît les stéréotypes culturels qu'un texte de fiction renferme) offre la forme rassurante du " déjà $v^{14}$ ", l'effet de stéréotypage dans le récit parodique postmoderne associe le « déjà vu » à l'anxiété de l'auteur (plutôt qu'à la rassurance du lecteur), car les mécanismes de l'ironie et du caricatural par le biais desquels la parodie opère, désignent surtout la crise et l'incertitude de l'écriture littéraire postmoderne ${ }^{15}$. 
11 Cependant, l'esthétisme consacré par le mode parodique ne pourrait pas prétendre pouvoir « immuniser » l'écriture romanesque contre les stéréotypes culturels : la tension entre la portée métafictionnelle du récit postmoderne et la possible persistance de certains stéréotypes culturels dépendrait largement de la forme narrative ou du sousgenre romanesque parodié. Ainsi, la version ironique du roman de voyage et du récit de la quête (le sous-genre et la forme narrative qui nous concernent dans le présent travail) ne pourrait guère dissoudre leur portée éthique dans l'esthétisme de la métafiction. Plusieurs critiques, comme Marie Louise Pratt et Véronique Magri, avaient déjà insisté sur les implications politiques et éthiques du roman de voyage. Quand Magri nous signale que la recherche de l'altérité est le noyau du récit de voyage, elle suggère que la 'narration' et la description de cette altérité sont souvent commandées par un stéréotypage potentiel ${ }^{16}$. C'est en partie ce potentiel là que la parodie du roman de voyage et l'esthétisation du récit de la quête que l'œuvre de John Barth a dû confronter à travers son jeu intertextuel et sa thématique métafictionnelle.

\section{L'orientation "esthétisante" comme résistance au stéréotypage du motif de la quête : The Floating Opera de John Barth}

Dans la fiction de John Barth, le motif récurrent du voyage, promu par le registre de la navigation, de l'aventure maritime, et subverti par le mode ironique de la répétition parodique, suggère la formulation progressive au sein de la poétique barthienne d'une catégorie fluide, presque insaisissable, qui vacille entre le jeu intertextuel (reconnaissant le statut archétypal des œuvres littéraires telles que l'odyssée ou Don Quichotte) et la mise à nu de l'aspect stéréotypé des motifs littéraires que le récit barthien s'approprie. En effet, la parodie soutenue du roman de voyage, du récit maritime, dans les récits tels que The Tidewater Tales ou The Last Voyage of Somebody the Sailor, dégage une ambiguiité caractéristique de la poétique postmoderne. D'une part, ces récits assument explicitement l'impossibilité de dépasser ou d'éclipser les textes canoniques parodiés. Par conséquent, c'est à l'autorité de ces textes que le récit parodique barthien fait appel ${ }^{17}$. D'autre part, l'ampleur de l'ironie subversive et de la dimension comique déstabilise l'image archétypale dont jouissent les classiques parodiés, et par suite rend incertain le ton de l'hommage attendu par le lecteur. En fait, la version parodique du roman de voyage et du motif de la quête que nous offre la fiction de Barth s'articule autour des stratégies narratives qui font écho aux mécanismes propres à la production des stéréotypes littéraires (condensation, schématisation, simplification de l'hypotexte), mais qui suggèrent aussi l'impossibilité de l'originalité (impossibilité d'aller au-delà de la répétition).

13 Toutefois, le vacillement entre la mise en évidence de l'aspect stéréotypé du motif de la quête dans les traditions romanesques et l'image archétypale de ces traditions littéraires parodiées ne revêt pas la même signification (quant à la poétique du roman postmoderne telle que l'auteur la conçoit) dans le premier roman de Barth et dans les récits écrits trente ans plus tard. Aussi, l'ironie subversive dans les romans de Barth où le voyage (notamment le voyage maritime) est un motif central, dévoile-t-elle l'épuisement de la symbolique classique de l'écriture du voyage (dans la tradition américaine) comme recherche de l'altérité ${ }^{18}$. Mais au lieu de transformer cette recherche de l'altérité 
(devenue cliché) en quête identitaire (elle aussi devenue stéréotype littéraire), le récit barthien met en évidence sa tentative de déjouer les deux axes de la symbolique conventionnelle de la quête, en donnant à ce motif une orientation substantiellement métafictionnelle, mais aussi, en mettant en question cette même orientation.

En effet, dans The Floating Opera $\left(1956^{19}\right)$, la subversion parodique du motif du voyage et de la symbolique de la navigation ébranle moins le leitmotiv de la quête que la linéarité présumée du récit narratif. Dans The Tidewater Tales (et même dans The Last Voyage of Sombody the Sailor), par contre, ce sont les présupposés du motif de la quête dans l'intrigue fictionnelle qui deviennent eux-mêmes la risée de la parodie barthienne.

Dans le premier roman de Barth, décrit par l'auteur comme «nihilist comedy [comédie nihiliste ${ }^{20}$ ] ", la métaphore de la navigation qui s'étend sur la totalité du récit, et la quête dans laquelle Todd Andrews s'engage, évoluent parallèlement l'une à l'autre, sans que l'intrigue ne parvienne à contenir la figure de style de manière conventionnelle. La tension entre l'aspect stylistique (l'image) et l'intrigue conventionnelle permet au récit de Barth de déjouer l'illusion de linéarité narrative en mettant en évidence la verticalité propre au mode poétique de l'écriture littéraire. La quête de Todd Andrews est menée à travers deux activités, à savoir l'écriture et la survie. Écrire prend la forme de la rédaction d'un texte désigné comme « the Inquiry [l'Enquête ] » et qui se présente comme le produit inachevé de plusieurs années d'efforts à essayer de comprendre le suicide inexpliqué de son père. L'activité de " vivre » acquiert sa signification du rituel qui consiste à payer au quotidien sa chambre d'hôtel. Le développement simultané de l'axe métafictionnel créé par les doubles du récit barthien (le texte de l'enquête - The Inquiry - et l'intrigue envisagée par Todd) et de l'histoire mimétique engendre une tension soutenue, joignant l'autocritique littéraire et une orientation prononcée vers l'esthétisme à une appropriation du récit de la quête dont l'autorité se trouve reconnue dans la subversion ironique même. La quête de Todd qui meuble l'axe mimétique du récit est cernée par cinq expériences majeures dont l'aboutissement de chacune d'entre elles est spécifié en termes de cinq découverte : la mort, la sexualité, l'impossibilité de l'amour, l'animalité de l'être humain et l'art. Les deux axes du récit de The Floating Opera s'articulent autour de la métaphore de la navigation dont l'image du bateau n'est qu'une variante.

En effet, le motif du bateau navigant vacille tout au long du récit entre son rôle de composante de l'intrigue (du contenu mimétique) faisant partie de son développement linéaire, et son rôle de double métaphorique du récit lui-même. Le narrateur identifie le récit (et l'œuvre même) au spectacle flottant qui a lieu à la fin de l'intrigue ${ }^{21}$. Une fois réduit à la métaphore du bateau-spectacle, le récit assume une dimension poétique (donc, verticale) qui éclipse la linéarité narrative. Le narrateur suggère même que les sens métaphoriques de l'opéra flottant débordent du texte de Barth (quand il énumère les êtres et les expériences qui «flottent », et où la métaphore communique le côté instable, voire insaisissable du sens de la vie ${ }^{22}$ ). Tout au long de The Floating Opera, l'axe métafictionnel promu par le miroitement interne, quand le spectacle flottant fonctionne comme le double métaphorique du récit fictionnel dans sa totalité, met continuellement en question le contenu mimétique afin de promouvoir la thématique autoréflexive de l'écriture romanesque. Cette thématique qui prône l'ouverture et la multiplicité du sens consacré par le mode métaphorique implique à la fois l'impossibilité de contrôler le sens et une conception d'un lecteur actif mais également malmené par le 'glissement' continu du sens. La métaphore de la navigation, détournée de son thème traditionnel de la quête identitaire ou existentielle, véhicule la thématique métafictionnelle et devient donc 
l'outil d'une autoréflexivité littéraire.Gerhard Hoffmann ${ }^{23}$ a déjà signalé, dans son commentaire de l'usage subversif des symboles dans la fiction postmoderne, le fait que dans l'œuvre de Barth le lieu de la navigation est la rivière et non pas l'océan ou la mer, et que son moyen n'est pas un navire mais un bateau en dérive, emporté au gré des vents.

Aussi, le bateau-spectacle comme double du récit, illustre-t-il un jeu esthétique que Barth désigne comme "regressus in infinitum». La régression du récit vers un solipsisme explicite est un jeu qui n'a cessé d'animer l'écriture romanesque postmoderne depuis que cette dernière a abandonné la chimère de l'originalitée ${ }^{24}$. Dans cette régression, la logique métonymique de l'intrigue annoncée se trouve pervertie par le mode métaphorique qui propulse vers l'avant-plan du texte le souci métafictionnel de l'autodéfinition et de l'autocritique. Le mouvement opposé, à savoir la progression engendrée par la quête (d'une vérité ou d'une réponse) d'un personnage secoué par une série de découvertes traumatisantes ${ }^{25}$ s'avère être contingent par rapport au développement de la métaphore centrale. L'image de la navigation tend à contenir l'intrigue mimétique plutôt qu'à l'illustrer. D'ailleurs, tout au long du récit de The Floating Opera, la signification du contenu mimétique est subordonnée au «parcours» de l'image du bateau, et par conséquent, le mode métonymique de l'écriture romanesque tel que David Lodge ${ }^{26}$ l'a défini, se trouve continuellement perverti par le mode métaphorique propre à la poésie. Le mode métaphorique qui opère par le biais de l'image de la navigation, ne fait pas qu'instaurer la discontinuité dans le récit barthien, il usurpe et s'approprie même la linéarité traditionnelle de l'intrigue.

18 Ainsi, la narration de l'aventure de Todd avec Jane Macks semble faire appel à la métaphore du bateau pour suggérer la découverte de Todd que l'amour est impossible. Mais en fait, l'irruption du mode métaphorique dès la première rencontre de Todd avec Jane, relie le personnage de Jane Macks au niveau symbolique du récit où elle acquiert sa signification dans l'«histoire» de la métaphore. L'aventure amoureuse de Todd et Jane est annoncée quand le mari de celle-ci la présente comme une île, ce que le narrateur confirme («She was indeed 'Ruxton and Gibson island' [Elle était, en effet, l'île de Ruxton et Gibson $\left.\left.{ }^{27}\right] »\right)$. Todd, à son tour, décrit Jane à travers des images qui l'associent à la navigation et aux bateaux ( « she kept reminding me of sailboats [elle n'a cessé de me faire penser aux voiliers $\left.\left.{ }^{28}\right] »\right)$. Plus précisément, le portrait de Jane est articulé dans le passage de "She was ... "Ruxton and Gibson island" " vers "she was in fact an ardent sailor [elle était en fait un ardent matelot $\left.{ }^{29}\right] »$, si bien que la métamorphose des images mène à la « résurrection » de la métaphore de la navigation.

Que le portrait changeant de Jane soit assimilé au mouvement vers cette " résurrection » de la métaphore, cela est justifié par le rôle du personnage féminin dans la quête de Todd: au début de leur rencontre, Jane est l'objet de son désir (ce qui explique son association avec l'image de l'île), mais devant l'impuissance de Todd, elle devient très vite l'agent de la quête quand elle mène leur aventure sexuelle (c'est pourquoi elle est associée à la métaphore du navigateur). Cependant, les images de l'île et du navigateur, présentées au lecteur avant l'évolution de la relation entre les deux personnages, deviennent le centre thématique à la lumière duquel l'aventure amoureuse va acquérir sa signification. L'expérience des personnages est déchiffrée à partir d'une perspective qui permettrait au lecteur de comprendre l'évolution des images de l'île, des bateaux navigants, vers la métaphore centrale du navigateur. En effet, le processus de la lecture est à même d'activer la subordination de l'intrigue linéaire à l'évolution de (et vers) la métaphore, puisqu'il se trouve guidé par le parcours « métonymique » attribué à l'image 
du navigateur. C'est précisément cette stratégie narrative consistant à subvertir la relation conventionnelle entre figure de style et contenu mimétique, qui distingue souvent l'écriture romanesque postmoderne et exprime sa tendance vers l'anti-réalisme et sa prédilection pour le mode poétique de l'écriture romanesque.

De même, l'expérience de la guerre qui permet à Todd de découvrir son "animalité» (quand il se décide à assassiner le soldat allemand en dépit de leur brève mais sincère amitié) est complètement subordonnée à la nouvelle signification qu'elle donne à la passion de Todd pour la construction des bateaux. Avant la guerre, cette passion est motivée par le rêve que le personnage nourrit de naviguer à travers le monde (le bateau est explicitement associé au désir de découvrir le monde et de se découvrir), mais la totalité du processus de la construction s'avère être maladroit. La découverte par Todd de l'animalité de l'être humain, n'a de répercussion que sur sa vision esthétique. Et la construction du bateau n'est plus motivée à ses yeux par le rêve d'aventure, elle devient une recherche de la perfection qui n'appartient qu'à l'art et non à la vie. Par conséquent, quand Todd renoue avec sa passion, le bateau dont il entreprend la construction, est conçu comme objet esthétique valorisé en lui-même.

Le bateau est soustrait à un type figé de quête dès lors qu'il devient le moyen d'une quête esthétique. Le motif du bateau devient l'élément d'une réflexion métafictionnelle sur le fondement de la tradition réaliste. Ainsi, l'orientation du récit barthien vers l'esthétisme est annoncée et justifiée par l'échec de la tradition humaniste qui a longtemps commandé le réalisme littéraire. Mais l'esprit d'auto-ironie consubstantiel à la fiction de Barth fait que le fossé se creuse progressivement entre l'œuvre « artisanale » de Todd Andrews et le spectacle artistique associé au bateau («l'opéra flottant»). Aussi, l'ébranlement de la conventionnelle linéarité narrative (une linéarité régie par le mode métonymique propre à la narrativité), est-il systématiquement subverti par la discontinuité propre au mode métaphorique et une pseudo-linéarité qui lui est attribuée quand le récit met en avantplan les métamorphoses de l'image centrale de la navigation. Cette stratégie narrative qui se présente souvent sous l'aspect d'un " tour de force ", lorsque la continuité est usurpée à l'intrigue fictionnelle conventionnelle pour être réinventée en pseudo-linéarité d'une métaphore centrale, est typique de l'écriture romanesque postmoderne. Néanmoins, dans le premier roman de Barth, la refonte des motifs de la quête et du voyage maritime dans la lignée de la métafiction et de l'esthétisme littéraire, ne semble pas remettre en question la thématique romanesque elle-même, ni impliquer l'impossibilité d'aller audelà des formes littéraires stéréotypées propres au roman (récit de voyage, roman de formation). C'est en revisitant cette même thématique trente ans plus tard que la poétique barthienne se trouve confrontée aux limites du récit de la quête et de la métafiction même.

\section{L'impossible quête ou stéréotyper l'aventure : The Tidewater Tales}

Dans ses récits publiés trente ans après The Floating Opera, John Barth relie de manière plus annoncée sa refonte du motif de la quête à la subversion parodique du roman de voyage, notamment le roman maritime. Mais le récit barthien dans The TideWater Tales ${ }^{30}$ est confronté également aux limites de la pure métafiction puisque celle-ci devient ellemême l'objet d'une réflexion critique. Le récit barthien ne se complaît plus dans la quête purement esthétique, et c'est d'ailleurs ce qui explique la description par certains 
critiques $^{31} \mathrm{du}$ retour de la fiction barthienne des années quatre-vingt au réalisme littéraire. Mais plutôt qu'un simple retour à la tradition réaliste, on perçoit dans The Tidewater Tales et The Last Voyage une volonté de s'attaquer aux limites de l'orientation métafictionnelle propre au roman postmoderne, une réflexion autocritique sur les risques de stéréotypage qu'engendre le confinement de l'écriture romanesque dans l'interminable jeu de la répétition ironique et auto-ironique.

Ainsi, dans The TideWater Tales les traits conventionnels du roman de voyage sont systématiquement suggérés, voire amplifiés pour être ensuite déconstruits par la mise à nu de leur qualité 'stéréotypée'. La répétition parodique du récit de l'aventure maritime, forme particulière du roman de voyage, permet à l'œuvre barthienne d'amplifier son aspect stéréotypé, mais aussi d'axer sa thématique sur sa propre tentative de déjouer cet aspect. D'où le miroitement interne du récit et sa vertigineuse spirale d'autoréflexivité. La visée encyclopédique du roman de voyage est réinventée dans The Tidewater Tales sous la forme d'un croisement interminable de récits où Peter et Katherine (le couple navigant) sont tour à tour narrateurs ou narrataires. La réitération par Peter, qui est le double de l'auteur et se prend pour l'écrivain même du roman, de son penchant pour l'écriture minimaliste (pour l'écriture de nouvelles où ne figurerait que l'essentiel) confine The Tidewater Tales dans une double négation ironique : le récit avec ses 656 pages est loin d'incarner le minimalisme prétendu, et sa spirale de ramifications et d'imbrications narratives qui finissent dans une forme d'autonégation, pervertit complètement la dimension encyclopédique (la notion de connaissance devient inenvisageable). Aussi, le huis clos du bateau qui meuble l'intrigue du roman maritime est-il à la fois reproduit (quand la narration nous rappelle les conditions de navigation du couple solitaire), et subverti par l'inhabituelle présence féminine (Katherine, dont la grossesse touche presque à son terme).

24 Le jeu entretenu par Peter et Katherine et qui consiste à raconter des histoires à tour de rôle (d'où la phrase «tell me a story » qui acquiert la fonction de refrain), ou à devenir les narrataires d'autres conteurs (Doug, Talbott), concrétise l'effort du récit à dépasser l'aspect stéréotypé du roman maritime afin de substantiver ce que certaines descriptions théoriques qualifient comme le risque de déclassement ou de basculement ${ }^{32}$ inhérent au sous-genre romanesque. En effet, l'oscillation systématique tout au long de The TideWater Tales entre le réalisme domestique, le récit de vie, le récit métafictionnel, le récit satirique, le roman d'espionnage, et le roman d'aventure maritime, met l'accent sur l'investissement délibéré des possibilités de "déclassement ». Le roman postmoderne tel que John Barth l'a conçu puise sa poétique dans ces possibilités de déstabiliser les stratifications et les frontières entre les formes littéraires, et de miser sur le potentiel qu'a l'acte narratif de faire basculer le récit entres diverses traditions littéraires. Ainsi le récit barthien ne se borne-t-il pas à donner un contenu métafictionnel au motif de la quête, il l'informe du risque même de basculement, en maintenant à l'avant-plan la limite ambiguë entre la consécration de l'aspect stéréotypé du récit maritime (du récit de la quête plus généralement) et le désir (souvent impossible) de subvertir ou de déjouer celui-ci.

Dans la fiction américaine, Herman Melville (1813-1891) avait déjà exploré le potentiel de déviation inhérent au roman maritime (dont il est le grand fondateur dans la littérature américaine), dans son dernier roman, Billy Budd ${ }^{33}$, où le narrateur qualifie sa digression de «literary sin» (le "péché littéraire » étant le double métafictionnel du thème de la culpabilité qui est au centre de l'intrigue). 
Dans The TideWater Tales, le motif du naufrage est à la fois amplifié et atténué. Au début de leur navigation, Peter et Katherine observent l'approche d'une tempête, et donc le narrateur suggère la possibilité du naufrage de leur bateau (p.81). Mais la dimension métafictionnelle est soudain propulsée à l'avant-plan du récit, de manière à interrompre d'abord l'intrigue conventionnelle, puis à problématiser l'ancrage du récit barthien dans le roman maritime traditionnel. L'annonce de la tempête est faite alors que Peter raconte à sa femmel'histoire qui est la leur. Peter fait allusion au caractère double de la tempête («twin storms ») pour conclure avec l'annonce du «book» qui en est le fruit ${ }^{34}$. Le jeu labyrinthique de miroitement narratif, établi dans toute l'œuvre de Barth, introduit un effet d'auto-ironie qui subvertit la vraisemblance sans complètement détruire l'intrigue, mais en prétendant la réduire au point zéro. Le livre annoncé par Peter et qui succède immédiatement à son commentaire, est en fait le nouveau commencement du récit. La scène du couple se battant à bord du bateau contre un vent violent inaugure le chapitre " Day 0 » (« le jour zéro » annonce un nouveau commencement du récit), et permet à l'idée du naufrage de continuer à planer. Mais le risque de naufrage n'est évoqué que pour nous rappeler à la fois un aspect stéréotypé du roman maritime et le désir de dépassement qui anime le récit barthien : ce n'est pas l'aboutissement d'un tel dépassement qui importe, mais le fait qu'il soit incorporé dans le récit comme un désir impossible, insaisissable (voire «non-présentable»). Le narrateur-protagoniste ne fait pas que s'autoproclamer écrivain du récit qu'il nous raconte, il dévoile sa conscience du caractère figé, prévisible de la situation de naufrage imminent dans laquelle il se trouve. C'est ainsi qu'en décrivant leur situation périlleuse au milieu des vagues violentes, Peter nous dit que comme toujours dans de telles circonstances sa femme et lui se demandent si les autres savaient quelque chose qu'eux mêmes auraient dû savoir ${ }^{35}$. Ce "quelque chose ", Peter le définit plus tard comme la convention littéraire qui fait que le personnage aventurier survive toujours aux risques ${ }^{36}$. Ce que Peter annonce comme situation stéréotypée propre à l'intrigue du récit fictionnel, se trouve déjoué par un tournant métafictionnel, ce qui démontre que Peter se trompe en se prenant pour l'auteur lui-même. Mais cela montre également que le récit barthien est acculé à progresser à la lumière de l'impossible dépassement des conventions et aspects propres au roman d'aventure maritime, une impossibilité que seul le « tournant » métafictionnel peut essayer de déjouer.

27 A la question que Katherine pose: «what are we doing here?» («que faisons-nous ici ?»), Peter fournit en effet la réponse qui réduit tout le motif du voyage maritime à la symbolique métafictionnelle : "a cruise through the ocean of Story. Since you're asking, that's what we are up to ${ }^{37}$ [une croisière à travers l'océan des histoires, puisque tu le demandes, c'est à quoi nous sommes dévolus]». Une telle réponse ne se limite pas à réduire le motif de la quête au niveau métafictionnel, autoréflexif, du récit, elle installe aussi un jeu énonciatif qui vacille entre l'affirmation de l'orientation " métaphorisante » du récit maritime conventionnel (une orientation qui a, elle-même, acquis un caractère stéréotypé, dés lors qu'elle s'est trouvée décrite comme sa "vocation profonde ${ }^{38}$ ") et l'ironie de l'autonégation qu'une telle orientation peut engendrer quand elle présente l'aventure et la quête comme l'apanage de la métafiction. Tout au long du récit de The TideWater Tales, le jeu énonciatif vacille entre un semblant de progression dans l'aventure maritime (par les descriptions récurrentes du bateau qui avance dans la baie) et une autonégation qui réduit l'intrigue au point zéro. L'auto- négation est l'aboutissement d'un jeu d'autoréflexivité qui donne à la conventionnelle dimension symbolique du roman maritime une orientation métafictionnelle. 
Ainsi, la scène du bateau malmené par le vent et du couple craignant le naufrage (139) est immédiatement déconstruite par un discours assez déroutant dans lequel Peter, s'adressant au lecteur, fait appel à son bon sens : « Peter aurait-il mis sa femme en danger et serait-il en train de concocter ce récit d'une certaine épaisseur s'il ne s'agissait pas d'autre chose que d'un instant d'amusement en attendant l'arrivée du docteur ${ }^{39}$ ? " La métalepse qui est ici à la fois intérieure et extérieure ${ }^{40}$ (car le récit brouille systématiquement la frontière entre mode homodiégétique et mode hétérodiégétique), fait de la transgression des frontières entre monde fictionnel et monde historique (celui du lecteur) un jeu d'autonégation narrative. Quand le récit de Tidewater reprend le motif de l'aventure maritime, ce n'est que pour tomber dans l'autodérision, surtout lorsque Katherine rappelle àson mari qu'ils ne peuvent pas prétendre confronter les dangers de l'océan, que leur aventure maritime ne dépasse point la baie de Chesapeake : « It is not as if we're in mid-Atlantic... Chesapeake Bay for Christ's sake : a Mickey Mouse crack in the coastline [Ce n'est pas comme si on était au milieu de l'Atlantique... Chesapeake Bay pour l'amour de Dieu, une fissure dans le littoral ${ }^{41}$ ]». La métaphore empruntée au monde de Walt Disney nous renvoie à cette «hyper-réalité » de la culture postmoderne que Jean Baudrillard ${ }^{42}$ associe à la prédominance du "simulacrum ", signalant ainsi le caractère artificiel du motif de l'aventure maritime. La négation ironique des connotations d'immensité et de l'infini, qui se trouve consolidée par le commentaire d'Irma (insistant sur le fait que l'aventure maritime de sa fille et son gendre ne devrait pas aller au delà de la piscine ${ }^{43}$ ), maintient dans le récit de Barth un lien indéniable entre auto- réflexivité littéraire et autodérision. Ce même effet d'autodérision se développe comme la conséquence directe du dévoilement de l'aspect figé et stéréotypé que semble revêtir la symbolique conventionnelle du roman maritime.

Dans The TideWater Tales, les commentaires de nature métacritique (et qui parfois dépassent le récit d'aventures maritimes pour porter sur les conventions de la fiction dans leur totalité) présentent l'intrigue du récit de fiction et le statut du personnage héroïque dans ce qu'ils ont de plus figé. Ainsi, ces commentaires propulsent dans le récit Barthien un stéréotypage qui renforce la tendance du récit à l'autodérision. Peter nous rappelle que dans le récit d'aventures le héros est toujours " préservé ", sauvé du danger, à l'opposé de la vie et son lot de morts absurdes ${ }^{44}$. La dimension d'auto-ironie générée par un tel commentaire se trouve concrétisée à travers le personnage de Katherine qui, enceinte de huit mois et demi, ne semble pas être "physiquement» affectée par les secousses violentes que subit le bateau sous l'effet du vent.

De tels gestes métafictionnels qui attirent l'attention sur l'artifice de la composition romanesque, semblent procurer au romancier, nous dit David Lodge, la liberté de brouiller les modes narratifs (chose dont profite amplement le récit barthien, surtout dans TideWater) et d'échapper à l'accusation d'être « de mauvaise foi ${ }^{45}$ ». Dans les récits de John Barth, l'auto-réfléxivité, le jeu de miroitement, signalent que cette accusation n'est évitée qu'au prix de l'autonégation. Aussi, l'autocritique littéraire, comme nous l'apprend l'œuvre barthienne elle-même, génère-t-elle un stéréotypage des motifs conventionnels de la composition romanesque. Une dimension significative d'ironie dans The TideWater Tales émane du lien substantiel établi tout au long du récit entre l'aboutissement de la réflexion métafictionnelle sur l'autodérision et le conséquent stéréotypage des aspects conventionnels de l'écriture romanesque.

31 Dans le récit de TideWater également, cette complicité entre autodérision et stéréotypage littéraire se trouve renforcée par le jeu intertextuel quand Peter et Katherine évoquent 
les textes littéraires archétypaux, de Huckleberry Finn (Mark Twain) à Arthur Gordon Pym (Edgar A. Poe), et de The Secret Agent (Joseph Conrad) à Moby Dick (Herman Melville). Ce sont surtout les deux récits canoniques de la quête, à savoir l'Odyssée et Don Quichotte, qui régissent le jeu intertextuel dans sa totalité et marquent son mouvement constant entre l'auto-dérision et l'auto-légitimation. En effet, l'intertextualité dans Tidewater commence par la comparaison et la citation et par leur effet indéniable d'auto-ironie (quand Peter se compare à Ulysse et même à Homère ${ }^{46}$ ), pour aboutir à l'appropriation (quand Barth récrit Don Quichotte, le texte qui demeure au niveau de « récit potentiel » pour Peter ${ }^{47}$ ).

La composante classique du roman de voyage qui fait que le protagoniste voyageur soit influencé par sa lecture des œuvres littéraires portant sur le voyage et l'errance ${ }^{48}$, est remodelée par le récit barthien de manière à rendre le jeu intertextuel plus vital, capable de confronter ses propres limites, notamment le risque de stéréotyper l'hypotexte et/ou l'hypertexte. En effet, l'appropriation de la thématique de la lecture littéraire dans The Tidewater est régie par un procédé plus complexe que le motif traditionnel de l'inspiration, ou de l'image de soi, que cette lecture traditionnellement procurait au personnage aventurier. La conscience et la « vocation » littéraires de Peter dans TideWater font de cette thématique de la lecture le moyen qui permet à Peter de découvrir que sa propre expérience du voyage est un cliché littéraire. En retraçant ses débuts de romancier qui remontent à un voyage depuis l'Europe vers l'Amérique, Peter identifie son expérience en termes de stéréotype littéraire, sans doute en faisant allusion à l'intrigue jamesienne et à son paradigme du personnage américain voyageant entre l'Europe et le nouveau monde. Souvent dans The Tidewater Tales, les citations et les évocations des récits canoniques de l'aventure et de la quête creusent le fossé entre les voix narratives (Peter et Katherine font de cette citation un jeu « innocent») et la voix auctoriale qui dévoile la conséquence de ce jeu de citations, à savoir le stéréotypage des personnages barthiens. Nicole Biagoli a suggéré, dans sa description de l'intertextualité qui émane de la « reprise fictionnelle ${ }^{49}$ ", que la citation attribuée au personnage ainsi que son potentiel représentatif peuvent soit éviter au personnage de tomber dans le stéréotype (grâce à l'appropriation), soit permettre au personnage d'émerger comme un stéréotype. L'appropriation dans l'œuvre de Barth dévoile la possibilité, voire l'imminence d'un basculement vers le stéréotype littéraire, un basculement qui (dans un texte foncièrement métafictionnel) guette le personnage citant.

Aussi, l'évocation répétitive de récits littéraires canoniques comme L'Odyssée, Don Quichotte, Moby Dick, ou Huckleberry Finn, est-elle informée par le désir impossible de dépassement : Peter, le double de l'auteur, invoque ces «monuments » littéraires pour en conclure que son ambition à lui (comme romancier) est de "composer une image plus vaste, plus riche que les livres qui déclencheraient sa naissance ${ }^{50}$ ». L'optimisme naïf du double de l'auteur l'empêche de voir que la répétition à laquelle est vouée la narrativité postmoderne génère une complicité indésirable entre écriture romanesque et stéréotypie.

C'est ainsi qu'en s'efforçant de se convaincre que le récit de son aventure maritime est doublement ancré dans l'Odyssée et Don Quichotte, Peter réduit, par un raisonnement simpliste, la signification des deux intertextes au croisement de l'élément marin et de l'élément terrestre: le détail de l'île dans l'œuvre de Cervantès (une œuvre que Peter qualifie de "dusty Don Quixote [Don Quichotte poussiéreux]») et l'aboutissement de l'aventure d'Ulysse sur la terre ferme. Cette interprétation simpliste ne manque pas de mettre en évidence le procédé par lequel le jeu intertextuel fige les textes archétypes 
invoqués en modèles stéréotypés. En réduisant les deux textes canoniques à un seul détail (le détail dont il a besoin pour justifier le double ancrage du récit de son aventure), Peter en fait des monuments figés. En outre, la réflexion critique sur le jeu intertextuel et ses risques renforce le brouillage de la frontière entre voix et point de vue narratifs (ceux de Peter) et voix auctoriale: le récit barthien ne prétend pas pouvoir échapper à l'ironie produite aux dépens du double de l'auteur. Au milieu de son commentaire comparatif de l'œuvre d'Homère et de celle de Cervantès, Peter se rappelle son voyage en Espagne et comment il a sillonné les routes (en "auto-stop »!) entre Grenade et Madrid, pour se comparer, enfin, à Don Quichotte lui-même. La vision simpliste qui soutient la comparaison (en voyageant sur les routes, Peter était semblable à Don Quichotte, alors que sa présente navigation l'assimile à Ulysse) génère un cas extrême de stéréotypage auquel le jeu de l'intertextualité peut soumettre les œuvres littéraires canoniques.

Au-delà des déductions naïves de Peter, son dilemme nous renvoie à celui de Barth : le jeu intertextuel qui informe son récit implique la reconnaissance de l'impossibilité pour le romancier postmoderne de dépasser ce qu'Homère et Cervantès avaient déjà inventé dans la thématique de la quête. Mais aussi, en "verbalisant " l'influence insurmontable de leurs œuvres et en concrétisant la répétition inévitable de Don Quichotte et de l'Odyssée, Barth les voit se transformer en stéréotypes littéraires. L'autre aspect du dilemme, c'est que le récit romanesque postmoderne n'est pas seulement défié par les monuments littéraires du passé, il l'est également par les contemporains et leurs imitations parodiques des mêmes archétypes. Peter nous dit que son mal d'inspiration est en partie dû au fait que dans la brèche de dix ans qui sépare sa production de la première partie et de la deuxième partie de son roman, des imitateurs avaient déjà surgi avec leurs propres «Don Quichottes»(« He writes no further... Stung by imitators who leaped with Quixotes of their own into the ten-year breach between Part One and Two of his novel $\left.{ }^{51} »\right)$.

Ainsi, le stéréotypage des textes canoniques n'est pas l'effet d'une seule écriture, c'est le produit de tout un âge (celui de la littérature postmoderne) qui se trouve acculé à une répétition interminable des œuvres classiques. Le récit barthien, néanmoins, s'engage vers les derniers chapitres, à affronter les limites de ce stéréotypage, à en faire un jeu narratif au travers duquel les motifs de la quête et de l'aventure dans Don Quichotte sont réitérés de manière à renforcer l'aspect comique de l'œuvre archétypale de Cervantès et l'ambiguité du statut de l'œuvre de Barth. Le récit de Tidewater reprend le roman de Cervantès au niveau de la scène ambiguë de la descente à la caverne de Montesinos où la limite entre le réel et le rêve est bafouée ${ }^{52}$. La reprise amplifie l'effet comique de la scène de la caverne dans le récit de Cervantès en développant une confusion délibérée entre le littéral et le figuratif et en inventant le lien entre cet épisode et celui où Sancho gouverne ce qui semblait être une île. L'errance de Don Quichotte barthien dans la caverne le mène jusqu'à la rivière où le bateau (dont le nom suggère sa continuité comique avec le cheval : « Rocinante II ») rend possible son initiation à l'expérience de la navigation dans laquelle il finit par échouer sur l'île gouvernée par Sancho Panza. Ainsi, la scène d'un Don Quichotte maladroit en train de ramer au milieu de la rivière, emporté par un courant qu'il ne peut maîtriser, permet au jeu intertextuel dans le récit barthien de capitaliser sur le caractère stéréotypé du motif de la navigation, de l'aventure maritime, en l'assimilant à une œuvre archétypale qui avait elle-même ancré la naissance de l'écriture romanesque dans la répétition comique et l'autodérision. fondée par Ulysse (p.487), un Don Quichotte ébloui par son apprentissage de la 
navigation, et ce même épisode s'achève avec la rencontre de Peter Sagamore et de Don Quichotte sur un port à Lisbonne (p.491), laquelle rencontre pousse Peter à déduire que lui aussi a probablement échoué au Portugal depuis un grand roman. Le jeu intertextuel dans Tidewater amplifie à l'extrême le procédé de l'autoréflexivité littéraire (le Don Quichotte barthien lui-même lit et admire l'œuvre de Cervantès, p.491) comme s'il testait les limites du stéréotypage que la répétition ironique des récits fictionnels canoniques risque d'engendrer. Si on suit la logique de la vision barthienne exposée dans l'un de ses essais, on peut déduire que pour le romancier lui-même ce risque de stéréotypage s'applique à l'écriture romanesque postmoderne. Quant à la figure de Don Quichotte, elle incarne avec trois autres (Schéhérazade, Ulysse, et Huckleberry Finn) une forme iconique qui déborde l'œuvre de Cervantès. Barth qualifie ces quatre figures d'«images transcendantes » à résonance mythique : on les reconnaît sans même avoir lu les textes qui les ont créées $^{53}$. L'appropriation du personnage de Cervantès pourrait, par conséquent, être lue comme une consécration de l'ambiguïté foncière qui informe les possibilités du genre romanesque à l'ère postmoderne : sa reproduction inéluctable des figures iconiques telles que Don Quichotte et Ulysse est à la fois une reconnaissance du risque de stéréotypage qu'implique à présent l'écriture romanesque, et une manière de déjouer ce risque à travers l'autoconscience et l'autocritique littéraires.

Cependant, l'ambiguïté générée par l'appropriation de l'une des «figures transcendantes » n'est pas purement esthétique, et le stéréotypage n'est pas un effet purement littéraire. C'est une œuvre de John Barth lui-même qui l'illustre. La réécriture de Schéhérazade à travers l'appropriation de l'un de «ses " récits dans The Last Voyage of Somebody the Sailor (1991), a suscité (de par sa date de publication qui a coïncidé avec la guerre du Golfe) des lectures ${ }^{54}$ qui ont mis l'accent sur un effet de stéréotypage culturel produit surtout par l'usage de l'anachronisme. Le récit de Last Voyage s'articule autour de la rencontre d'un journaliste américain, Simon Behler, appartenant au vingtième siècle, et du Sinbad des Mille et Une Nuits. Leurs narrations juxtaposées de leurs aventures maritimes virent, par moments, aux commentaires sur les cultures respectives des deux personnages. Ainsi, le médiéval Sinbad, commentant les prouesses technologiques et scientifiques de l'Occident du vingtième siècle, profère des propos qui renforcent une image stéréotypée (et amplement médiatisée) de la culture arabo-musulmane comme violente et anti-moderne.

L'ambiguïté qui émane de la coexistence des signes évidents d'un stéréotypage culturel (qui aurait assuré peut-être le succès populaire du roman) avec une vision esthétique et " esthétisante ${ }^{55}$ " propulse le lecteur de The Last Voyage of Somebody the Sailor sur un terrain glissant où le dessein de l'auteur se trouve déjoué par les limites de son autorité (voire de sa légitimité) sur l'articulation du sens. La question du déchiffrage des stéréotypes (littéraires et/ou culturels) qui implique uneoscillation systématique entre l'interprétation et le jugement pose également la question de l'autorité du lectorat.

\section{Conclusion}

En conjuguant la perversion ironique du motif de la quête et la parodie du roman de voyage maritime l'œuvre de John Barth finit par soumettre à la réflexion critique l'orientation métafictionnelle même du roman postmoderne. Cette critique (qui prend la forme d'une auto-critique, voire d'une autodérision) accule le récit barthien à s'attaquer 
au spectre de la stéréotypie, ce qui finit à son tour par dévoiler les limites de l'autoréflexivité littéraire comme garante de la survie du genre romanesque.

Du reste, l'appropriation des textes canoniques tels que l'Odyssée ou Don Quichotte, qui s'opère dans le récit barthien (et plus généralementdans le roman postmoderne) dans un esprit d'auto-ironie, semble sauver le paradigme de la quête d'une liquidation inévitable, mais seulement au risque d'étendre l'effet de stéréotypie à ces textes archétypaux.

Aussi, le jeu intertextuel qui dévoile la tendance du récit parodique barthien à s'approprierl'altéritécomme étant une entité purement esthétique, ne semble pas soustraire le roman maritime (même dans sa version parodique) au risque d'un stéréotypage culturel que présentent généralement les récits de voyage ${ }^{56}$. L'œuvre barthienne nous montre qu'au-delà du souci esthétique (et « esthétisant»), l'auto-ironie qui régit le jeu intertextuel et le mode parodique constitue moins une issue définitive pour un roman dont la survie demeure incertaine, qu'une manière de multiplier les possibilités de stéréotypie au sein de l'écriture romanesque postmoderne.

\section{BIBLIOGRAPHIE}

AMOSSY RUTH, « Stereotypes and Representation in Fiction», Poetics Today, vol.5, n 4, 1984, pp. 689- 700.

BAKHTIN MIKHAÏL.The Dialogic Imagination, traduction de Caryl Emerson et Michael Holquist, Austin, University of Texas Press, 1981.

BARTH JOHN, The Floating Operaand The End of the Road [1956], New York, Anchor Books, 1988.

BARTH JOHN, « The Literature of Exhaustion », Atlantic Monthly, vol. 220, n², 1967, pp.29- 34.

BARTH JOHN, The TideWater Tales : A Novel, Baltimore, John Hopkins University Press, 1987.

BARTH JOHN, The Last Voyage of Somebody the Sailor, New York et Boston, Houghton Mifflin, 1991.

BARTH JOHN, Further Fridays. Essays, Lectures, and other Nonfiction, 1984-1994, Boston et New York, Little Brown \&Co., 1995.

BIAGIOLI NICOLE, « Narration et intertextualité, une tentative de (ré)conciliation », Cahiers de Narratologie, $\mathrm{n}^{\circ} 13,8$ /09/ 2006

(http://revel.unice.fr/cnarra/document.html?id=314)

BLANTON CASEY, Travel Writing. The Self and the World [Écriture de voyage. Le Moi et le monde], Londres, Routledge, 1995, 2002.

BOYER ALAN-MICHEL, " Pour une typologie du roman d'aventures maritimes ", in P. Masson et M. Blain (eds.), Rêveries marines et formes littéraires, Nantes, Université de Nantes, Pleins Feux, 2001, pp.60- 75 .

CARMICHAEL THOMAS, " Postmodernism Reconsidered : The Return of the Real in John Barth's Sabbatical and The Tidewater Tales ", Revue Française d'Etudes Américaines n62, Novembre 1994, pp. 329- 338. 
CHARTIER PIERRE, Introduction aux grandes théories du roman, Paris, Dunod Editions, 1996.

COHN DORRIT, «Métalepse et mise en abyme», in J. Pier et J.M. Schaeffer (eds.), Métalepses. Entorses au pacte de la représentation, Paris, Éditions de l'EHESS, 2005, pp.122- 130.

DUMBRAVA GABRIELA, « From Archetype to Stereotype : a Postmodern Re-reading of the South », in European Journal of American Studies Janvier 2007-2, 08/01/2008.

(http://ejas.revues.org/document1693.html)

GANNIER ODILE, «Stéréotypes et roman maritime : gros temps sur la Sea Trilogy. To the Ends of the Earth (trilogie maritime de William Golding) », Loxias n¹7, 28/01/ 2007, (http://revel.unice.fr/loxias/document.html)

HADDOX THOMAS F, « The Floating Opera and Southern Modernism of the 1950s [The Floating Opera et le modernisme du sud des années 50] », Twentieth-Century Literature, vol.54, $\mathrm{n}^{\circ} 3$, Automne 2008, pp. 307-338.

HASSAN IHAB, "Quest : Forms of Adventure in Contemporary American Literature " ["Quête : Formes de l'aventure dans la literature américaine contemporaine"], in Malcolm Bradbury et Sigmond Ro (eds.), Contemporary AmericanFiction, Londres, Edward Arnold, 1987, pp. 123- 137.

HOFFMANN GERHARD, From Modernism to Postmodernism. Concepts and Strategies of Postmodern American Fiction [Du modernisme au postmodernisme. Concepts et stratégies de la fiction américaine postmoderne], New York, Éditions Rodopi, 2005.

HUTCHEON LINDA, A Theory of Parody. The Teachings of Twentieth-Century Art Forms [Théorie de la parodie. Les enseignements des formes artistiques du vingtième siècle], Londres et New York, Routledge, 1985.

JIANPING WANG, «Imagining Iraq and the cultural politics of misreading : John Barth's The Last Voyage of Somebody the Sailor» ["Imaginant l'Iraq et la politique culturelle de la mésinterprétation: The Last Voyage of Somebody the Sailor de John Barth], Journal of American Studies of Turkey, $\mathrm{n}^{\circ} 21,2005$, pp. 27-40.

LODGE DAVID, The Modes of Modern Writing. Metaphor, Metonymy and the Typology of Modern Literature [Les modes de l'écriture moderne. Métaphore, métonymie et la typologie de la littérature moderne], Londres, Edward Arnold, 1977, pp.73-124.

LODGE DAVID, Consciousness and the Novel. Connected Essays [La Conscience et le roman], Londres, Penguin, 2002.

MAGRI VERONIQUE, «La description dans le récit de voyage », Cahiers de Narratologie : « Mélanges, espace et temps ", $\mathrm{n}^{\circ}$ 7, 1995-96, pp.35-48.

MAGRI VERONIQUE, « Du récit de voyage à la nouvelle : l'exemple de Maupassant : Au Soleil, Marroca, Mohammed-Fripouille, Un Soir, Allouma », inMarie-Christine Gomez-Géraud et Philippe Antoinne (eds.), Roman et récit de voyage, Paris, Presses de l'Université de Paris-Sorbonne, 2001, pp.155- 166 .

PAVEL THOMAS, La pensée du roman, Paris, Gallimard, 2003.

PRATT MARY-LOUISE. « Travel Narrative and Imperialist Vision [Récit de voyage et vision impérialiste] », in James Phelan et Peter Rabinowitz (eds.), Understanding Narrative, Columbus, Ohio State University Press, 1994, pp.199-221.

VENAYRE SYLVAIN, « Roman, aventure et histoire. La question de la vérité dans les récits d'aventures vécues ", in ID., Roman et récit de voyage, Paris, Presses de l'Université de ParisSorbonne, 2001, pp.67- 79 


\section{NOTES}

1. Voir l'étude de Harold Bloom, The Anxiety of Influence. A Theory of Poetry [1971], New York, Oxford University Press, 1997, p.8. Bien qu'elle se limite au cas des poètes, son analyse s'applique également à l'écriture romanesque.

2. "The Literature of Exhaustion », The Atlantic Monthly 220, n², 1967, pp.29- 34.

3. Voir par exemple : Gabriela Dumbrava, «From Archetype to Stereotype : a Postmodern Rereading of the South », in European Journal of American Studies Janvier 2007-2, 8/01/2008.

(http ://ejas.revues.org/document1693.html)

4. Pierre Chartier, Introduction aux grandes théories du roman, Paris, Dunod Editions, 1996, p.95.

5. Ibid, p.123.

6. Ian Watt dans The Rise of the Novel, Berkeley \& Los Angeles, University of California Press, 1967, déduit de sa brève allusion à la typification (qui se fait à travers la nomination) des personnages, que le rôle secondaire attribué au personnage type, en opposition au personnage principal qui, lui, est individualisé, signale un souci (surtout chez Fielding, le premier théoricien Anglais du roman) d'équilibre entre illusion réaliste et continuité avec les traditions littéraires classiques ( $\mathrm{p}$. $18,19,20)$.

7. Thomas Pavel, La pensée du roman, Paris, Gallimard, 2003, p.39.

8. Mikhail Bakhtin, The Dialogic Imagination, trad. Caryl Emerson et Michael Holquist, Austin, University of Texas Press, 1981, p.60, 301, 309

9. Pour la description du rôle du carnavalesque dans l'évolution du roman et son lien étroit avec la parodie, voir Bakhtin, op.cit., pp. 79-80, et pour l'interaction de la satire avec la parodie dans la production des stéréotypes, p.81.

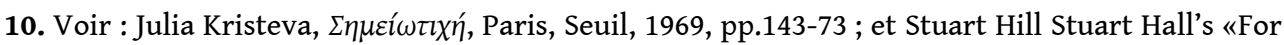
Allon White : Metaphors of Transformation», introduction à Carnival, Hysteria, and Writing par Allon White, Oxford, Clarendon Press, 1993, pp.6-7.

11. «Stereotypes and Representation in Fiction », Poetics Today, vol. 5, n4 4, 1984, p.689.

12. Mais, d'après Amossy, les modèles codifiés dont le stéréotype atteste l'existence, ne se limitent pas aux conventions littéraires; ils renferment surtout les formes culturelles liées aux croyances et à l'opinion publique(Ibid.), pp.689-90.

13. Ibid., p. 693.

14. Amossy, «Stereotypes and Representation... », op.cit., p.695.

15. Cette distinction est toutefois relative : l'acte de lecture est lui-même régi par des facteurs «non-littéraires». Ainsi, la parodie du personnage de Shéhérazade dans plusieurs récits barthiens a été interprétée dans une perspective métafictionnelle, alors que la version ironique du personnage de Sinbad que Barth nous offre dans The Last Voyage of Somebody the Sailor a suscité une polémique de part le contexte politique de sa publication, comme nous le verrons dans la dernière partie de ce travail.

16. Voir : "La description dans le récit de voyage", in Mélanges, espace et temps, Cahiers de Narratologie $n^{\circ} 7,1995-96, p .45$, où Magri insiste que dans le récit de voyage, la description «ne peut [...] se départir d'un effet de réduction, de schématisation, la description glissant vers le stéréotype.» Voir aussi : "Du récit de voyage à la nouvelle : l'exemple de Maupassant... in M. Gomez-Géraud et Ph. Antoinne (eds.), Roman et récit de voyage, Paris, Université de ParisSorbonne, 2001, p.161, où Magri signale que c'est la règle de l'exemplarité qui régit la sélection des fragments relatés, par conséquent, la lecture inductive que cette règle invite, renferme un potentiel de stéréotypage.

17. Voir : Linda Hutcheon dont A Theory of Parody, Londres, Routledge, 1985 formule le paradoxe de la parodie en termes de « authorized transgression». 
18. Voir : Casey Blanton, Travel Writing. The Self and the World, Routledge, 1995, 2002, pp. 17-18 en ce qui concerne la discussion de l'orientation des écritures de voyage dans la tradition américaine vers la recherche de l'altérité, et comment elles sont axées sur la quête identitaire dans la tradition européenne.

19. The Floating Opera and The End of the Road [1956], New York, Anchor Books, 1988.

20. John Barth, «Four Forwards. The Floating Opera and The End of the Road ", in Further Fridays. Essays, Lectures, and other Nonfiction, 1984-1994, Boston et New York, Little, Brown \&Co., 1995, p. 259.

21. "The floating Opera was tied up at Long Wharf...and some of this book happens aboard it. That's reason enough to use it as title. But there's a better reason. It always seemed a fine idea to me to build a showboat... and to keep a play going continuously. The boat wouldn't be moored, but would drift up and down the river on the tide, and the audience...could catch whatever part of the plot happened to unfold as the boar floated past..." p.7.

22. The Floating Opera, p.17.

23. Gerhard Hoffmann, From Modernism to Postmodernism. Concepts and Strategies of Postmodern American Fiction, New York, Éditions Rodopi, 2005, p.151.

24. Dans une étude récente, Thomas Haddox voit dans l'orientation métafictionnelle du premier roman de Barth l'expression d'un désir de fuir l'influence du « modernisme sudiste » incarné par William Faulkner et pressenti par le jeune romancier comme «menacent ", voir: "The Floating Opera and Southern Modernism of the 1950s ", Twentieth-Century Literature, vol. 54, n 3, Automne 2008, pp. 318- 320.

25. De la découverte du corps de son père au sous-sol de la maison parentale et de l'idée, voire la possibilité, de suicide, à son impuissance sexuelle, en passant par la découverte de l'animalité de l'homme.

26. David Lodge, The Modes of Modern Writing. Metaphor, Metonymy and the Typology of Modern Literature, London : Edward Arnold, 1977, pp.73-124. Il importe de noter ici que la dichotomie/ l'opposition binaire jackobsienne que Lodge généralise dans sa description des modes d'écriture romanesque et poétique a perdu de sa teneur depuis que les approches cognitives ont démontré que les même processus cognitifs de structuration et de projections sont aussi bien l'apanage de la métaphore que de la métonymie. Cependant, cette même distinction entre "mode métaphorique et «mode métonymique » d'écriture serait toujours valide dans la description des procédés qui permettent au récit métafictionnel de pervertir la linéarité narrative à travers l'usage de la métaphore.

27. The Floating Opera, p.24.

28. Ibid.

29. Ibid., p.25.

30. The Tidewater Tales : A Novel, Baltimore et Londres, John Hopkins University Press, 1987.

31. Voir par exemple : Thomas Carmichael, «Postmodernism Reconsidered : The Return of the Real in John Barth's Sabbatical and The Tidewater Tales ", in Revue Française d'Etudes Américaines n 62 (Novembre 1994), pp. 331-32.

32. Voir : Alan-Michel Boyer, « Pour une typologie du roman d'aventures maritimes », in Rêveries marines et formes littéraires, P. Masson et M. Blain (eds.), Nantes, Université de Nantes, Pleins Feux, 2001, p. 68, 69.

33. Herman Melville, Billy Budd, publiée à titre posthume, 1924, Milan, La Spiga Languages, 1995, p. 18.

34. The Tidewater Tales, p.82.

35. Ibid., pp.89-90.

36. Ibid., p. 141.

37. Ibid., p. 90.

38. Voir : Alan-Michel Boyer, « Pour une typologie du roman d'aventures maritimes », pp.66-67. 
39. "Our man is no boy; would he ever imaginably have put his wife and our all-but- delivered family at risk [...] would we be spinning out a book of some size thereabout, called The Tidewater Tales : A Novel-if this were merely one instance more of restless richies amusing themselves until the doctor comes?» (140).

40. Voir : Dorrit Cohen, «métalepses et mise en abyme », in Métalepses, J. Pier et J.M. Schaeffer (eds.), Paris, Éditions de l'EHESS, 2005, pp.122- 126.

41. Tidewater Tales, p.135.

42. Jean Baudrillard, Simulations, New York, Columbia University, coll. «Semiotext(e)», 1983, pp.23- 26.

43. Tidewater Tales, p.138.

44. Ibid., p. 141.

45. L'expression de Maurice Blanchot est reprise par David Lodge dans Consciousness and the Novel. Connected Essays, Penguin, 2002, p.81.

46. Tidewater Tales, p. 67, 93.

47. Ibid., p. 472- 477.

48. Voir : Sylvain Venayre, «Roman, aventure et histoire. La question de la vérité dans les récits d'aventures vécues », in M. Ch. Gomèz-Géraud et Ph. Antoine (eds.), Roman et récit de voyage, op.cit .,p.70.

49. Nicole Biagioli, "Narration et intertextualité, une tentative de (ré)conciliation », Nouvelle Approches de l'intertextualité, Cahiers de Narratologie, №13, 8/09/ 2006

(http ://revel.unice.fr/cnarra/document.html?id=314)

50. «... writerly ambition is to conjure an image larger and richer than any books worth of sentences that sets it forth...» (Tidewater Tales, 317).

51. Ibid., p. 345.

52. Miguel de Cervantes Saavedra, Don Quixote, Hertfordshire, Wordsworth Editions, 1993, 2ème partie, chapitre XXII, p. 496.

53. John Barth, «The Limits of Imagination », in Further Fridays, op.cit., p. 61.

54. Voir : Wang Jianping, «Imagining Iraq and the Cultural Politics of Misreading : John Barth's The Last Voyage of Somebody the Sailor », in Journal of American Studies of Turkey, n²1, 2005, p.28, 30, 36.

55. Dans The Friday Book. Essays and Other Nonfiction, Baltimore, The John Hopkins University Press, 1984, p.258 insiste sur ce qu'il appelle «l'intérêt professionnel » qu'il a comme conteur pour une autre conteur (Schéhérazade), dissociant son appropriation parodique et fréquente des Mille et une nuits des visions stéréotypées de «l'orientalisme...déploré par Edward Saïd... »

56. Voir : Magri, Véronique. «La description dans le récit de voyage », in Mélanges, espace et temps , Cahiers de Narratologie n 7, Université de Nice-Sophia Antipolis, 1995-96, pp.45- 47.

\section{RÉSUMÉS}

$\mathrm{Au}$ cours de ses mutations, la fiction de John Barth a incarné la vision esthétique propre au roman postmoderne américain, mais elle a également mis en question l'option métafictionnelle qui anime cette même vision. En tant que mode littéraire consubstantiel à la poétique barthienne, la parodie consacre dans maints récits la subversion systématique du motif de la quête en incorporant cette subversion à la déconstruction du roman de voyage. Le texte barthien 
révèle en effet que, loin de se borner à inscrire simplement le paradigme de la quête dans une orientation métafictionnelle, le romancier expérimente, dans un esprit d'autocritique, le risque de stéréotypage qu'implique la répétition ironique du motif littéraire et des textes canoniques parodiés.

In its incarnation of the self-critical, self-ironic spirit that informs the aesthetic vision of the postmodern novel, the fiction of John Barth has encompassed the inevitable questioning of the metafictional orientation itself. The mode of parody which is substantial to the Barthian poetics integrates the systematic subversion of the quest motif within a deliberate deconstruction of the traditional travel narrative. But, rather than confining the paradigm of the quest in a purely metafictional orientation, Barth's narratives involve a self-critical confrontation of the risk of stereotyping that the ironic repetition of the literary motif and of the canonical travel narratives implicate.

INDEX

Mots-clés : fiction, métafiction, Barth John, roman de voyage, parodie, intertextualité, quête, intrigue, poétique postmoderne, stéréotype

Index géographique : États-Unis

\section{AUTEUR}

\section{SALWA KAROUI-ELOUNELLI}

Maître-Assistante de littérature anglo-américaine à l'Université de Sousse (Tunisie) 